\title{
UM ESTUDO SOBRE PRÁTICAS DE LEITURA DE ALUNOS DO ENSINO MÉDIO
}

\author{
A Study about Reading Practices of High School Students
}

\author{
Paula Kracker FRANCESCON, UEL ${ }^{1}$
}

\begin{abstract}
RESUMO: Este trabalho apresenta os resultados parciais de pesquisa de cunho etnográfico que visa a descrever processos de leitura em língua estrangeira de um grupo de alunos do ensino Médio de uma escola pública de Londrina - PR. A coleta dos dados desta pesquisa ocorreu durante curso de leitura em língua inglesa oferecido aos alunos do ensino Médio da referida escola, ministrado pela professora-pesquisadora e com participação voluntária dos alunos. As atividades que proporcionaram a coleta dos dados foram produzidas com o objetivo de possibilitar leitura crítica aos participantes do curso. Assim, este estudo parte dos pressupostos dos Estudos Críticos da Linguagem (FAIRCLOUGH, 1989; 1992) e concepções sobre leitura crítica (WALLACE, 1992; FIGUEIREDO, 2000; HEBERLE, 2000). Neste trabalho, analiso dados provenientes de uma atividade de leitura realizada durante as aulas, com propósito de notar respostas críticas construídas pelos alunos para essa atividade específica. Com isso, é possível perceber o desenvolvimento das práticas de leitura desse grupo de alunos do ensino Médio.
\end{abstract}

PALAVRAS-CHAVE: Leitura Crítica; Língua Inglesa; Ensino Médio.

ABSTRACT: This research presents the partial results of an ethnographic research which aims to describe the reading processes in foreign language of a group of high school students from a public school in Londrina - PR. The data for this research was collected during reading classes offered to the students, conducted by the researcher and with voluntary participation of the students. The activities used during the classes, which provided the data to this work, were created with the objective of making it possible for the students to develop critical reading. So, this work uses the assumptions of Critical Language Studies (FAIRCLOUGH, 1989; 1992) and the conceptions about Critical Reading (WALLACE, 1992; FIGUEIREDO, 2000; HEBERLE, 2000). In this work, I analyze data provided by one reading activity performed during the classes, aiming at noticing critical answers construed by the students to this specific activity. From this analysis, it is possible to notice the development of reading practices of this group of high school students.

KEY-WORDS: Critical Reading; English Language; High School.

\footnotetext{
${ }^{1}$ Mestranda em Estudos da Linguagem pela UEL.
} 


\section{INTRODUÇÃO}

Este trabalho nasceu de meu interesse em investigar concepções que auxiliem no ensino de língua inglesa. Assim, nesta pesquisa, utilizo pressupostos de leitura crítica para desenvolver atividades de um curso de leitura em língua inglesa que ofertei a alunos do ensino Médio de uma escola pública de Londrina - PR. A escolha desse aparato teórico se justifica por ele contribuir para aspectos culturais e sociais na educação, buscando desenvolver consciência crítica com os alunos.

Dessa forma, o objetivo deste trabalho é descrever práticas de leitura em língua inglesa dos alunos do ensino Médio e, principalmente, analisar se eles constroem interpretação crítica dos textos que lêem. Mesmo considerando que os resultados apresentados neste estudo são parte de processo de aprendizagem mais longo (curso de leitura em língua inglesa), o foco deste trabalho é a participação de leitura dos alunos em uma atividade específica realizada durante as aulas.

\section{LEITURA CRÍTICA}

Estudos realizados na área da leitura conduzem ao modelo interativo, o qual propõe que a leitura deve ser realizada a partir de interação do leitor com o texto, com o autor e considerando o contexto no qual o texto se insere, buscando significados para o que está sendo lido por meio de diferentes fontes, como o próprio texto, o conhecimento prévio do leitor, o conhecimento do leitor sobre as condições de produção e recepção do texto. Esse modelo de leitura engloba duas formas de processamento textual: o topdown e o bottom-up ${ }^{2}$ (DECHANT, 1991; HARRISON, 2004).

Mais especificamente, além da leitura como o modelo interativo prevê, este estudo abarca visão de leitura crítica. Para iniciar a compreensão dessa concepção de leitura, começarei com observações acerca dos Estudos Críticos da Linguagem.

Fairclough (1989) defende que o ensino escolar deve ser pautado em visão crítica da linguagem. Isso significaria "mostrar conexões que podem estar escondidas das pessoas" (FAIRCLOUGH, 1989, p. 5). Essas conexões se referem à ligação entre

\footnotetext{
${ }^{2}$ O processamento textual top-down propõe que a leitura deve ser feita a partir do contexto e dos conhecimentos do leitor para o texto, referindo a uma atividade ativa de leitura. O modelo bottom-up refere-se à leitura que parte do texto, decodificando-o das menores para as maiores unidades textuais, ou seja, é uma atividade mais passiva, mas fundamental para qualquer outra concepção de leitura.
} 
linguagem, poder e ideologia. A linguagem é uma das práticas sociais onde o poder, por meio das ideologias vigentes, se manifesta e se perpetua. As ideologias se constituem nas convenções sociais. O que se entende como senso comum, comportamentos familiares, etc. são estabelecidos pelas convenções, o que faz com que não sejam questionados ou que as pessoas que agem de determinada maneira nem mesmo estejam conscientes de o fazerem, já que está dentro do comportamento comum, considerado como normal e não questionável dentro das regras que regem a sociedade da qual fazem parte.

Devido a relação entre linguagem e poder, Fairclough (1989) aponta a importância de a educação linguística visar a desenvolver consciência crítica da linguagem, de como o discurso funciona dentro das relações sociais em que se insere. $\mathrm{O}$ ensino de língua que busque formar essa consciência crítica da linguagem tem como seus propósitos desenvolver

\footnotetext{
uma consciência de como o discurso figura dentro das prática sociais, uma consciência que qualquer conhecimento de uma esfera da vida social é constituído como um discurso entre uma variedade de discursos co-existentes e concebíveis, que discursos diferentes são associados com perspectivas diferentes na esfera em questão e interesses diferentes, uma consciência de como os discursos podem funcionar ideologicamente nas relações sociais de poder, e assim por diante. É na base dessas compreensões de como o discurso funciona dentro das práticas sociais que as pessoas podem questionar e ver além dos discursos existentes, ou relações de dominação e marginalização existentes entre os discursos (FAIRCLOUGH, 1999, p. 74 - 75).
}

De acordo com essa visão, a partir da consciência crítica da linguagem, será possível desvendar as ideologias predominantes nos discursos e facilitar a prática emancipatória, podendo auxiliar grupos oprimidos a desafiar e transformar as práticas sociais dominantes (FAIRCLOUGH, 1989).

Nessa concepção de educação linguística, leitura crítica é parte fundamental. Esta pressupõe atitude ativa em relação ao texto por parte do leitor, e não a aceitação passiva das informações expostas pelo texto. Essa atitude ativa do leitor retoma a visão interativa de leitura, pois espera que o leitor faça relações entre o que está lendo com representações contidas em sua memória, seu conhecimento de mundo (FAIRCLOUGH, 1989; TOMITCH, 2000).

Tendo essa atitude ativa como ponto de partida, a leitura crítica leva o leitor ao questionamento das informações textuais, o que torna possível observação das 
convenções ideológicas e contestação das mesmas. Taglieber (2000, p. 16) apresenta características do conceito de leitura crítica, como

\begin{abstract}
meio de compreender a história e cultura de uma pessoa na estrutura social atual [...] e de promover um ativismo em direção a uma participação igual em todas as decisões que afetam e controlam nossas vidas. Processo interativo que usa vários níveis de pensamento simultaneamente (análise, síntese e avaliação), estabelecendo padrões e julgando ideias contra os padrões para verificar sua razoabilidade.
\end{abstract}

Como já dito anteriormente em relação aos Estudos Críticos da Linguagem, o reconhecimento e questionamento das convenções sociais, estabelecidas pelas ideologias dominantes e que se manifestam por meio da linguagem, levam à criação de consciência, empoderamento dos indivíduos em busca de sua emancipação, conscientização sobre as relações de poder existentes na sociedade (FAIRCLOUGH, 1989; FIGUEIREDO, 2000).

Assim, essa prática espera que, por meio da leitura crítica, o leitor será equipado discursivamente para ser capaz de reconstruir os significados do texto, aceitando ou questionando, desafiando a ideologia presente no texto. Figueiredo (2000) aponta que isso também pode ser realizado na leitura em língua estrangeira, já que o leitor também poderá se apoderar de conhecimentos de outras culturas, contribuindo para sua formação crítica. Da mesma maneira, Wallace (1992, p. 80) considera que o trabalho com a leitura em aulas de línguas estrangeiras pode

ajudar os leitores em inglês como língua estrangeira a se sentirem mais confiantes em tomar posições mais assertivas contra o texto, encorajá-los a sentirem que eles têm opções na maneira em que eles escolhem ler os textos, e ajudá-los a se sentirem em uma relação mais igual com o escritor.

\title{
METODOLOGIA
}

Neste momento, trato dos encaminhamentos metodológicos que nortearam os procedimentos desta pesquisa. Assim, discorrerei sobre a natureza, o contexto e os participantes da pesquisa e apresentarei a forma como a coleta de dados foi realizada. 


\section{NATUREZA DA PESQUISA}

Nesta pesquisa de cunho qualitativo, tomo os postulados do pós-positivismo como enquadramento paradigmático. Paradigma é definido por Guba (1990, p. 17) como "um conjunto básico de crenças que guia a ação, seja ela da variedade do dia-adia ou a ação tomada conforme uma investigação disciplinada". Dentro desse paradigma, defino a epistemologia como interpretativista e uso procedimentos metodológicos etnográficos.

Para a instância epistemológica norteadora da pesquisa, sigo concepções do interpretavismo, uma vez que ele sugere compreensão do subjetivo de uma forma relativamente objetiva. De acordo com essa postura, para compreender uma ação humana, é preciso entendê-la conforme os significados que os atores (os praticantes da ação) atribuem a ela. Dessa forma, o pesquisador adota atitude objetiva, pois deve relatar e compreender seu objeto de estudo da maneira como o observa, procurando se distanciar e não interferir na compreensão com suas crenças, teorias e preconceitos. Assim, é importante observar que o pesquisador vai a campo com o objetivo de aprender algo sobre seu objeto de estudo (os significados da ação humana) (SHWANDT, 2006).

Ainda relacionado ao interpretativismo, mais uma consideração é relevante: a de que as ações humanas ocorrem em um determinado espaço e tempo e esse contexto deve ser considerado pelo pesquisador ao formular sua descrição e interpretação do objeto de estudo. É por isso que o pesquisador deve considerar todo o contexto de realização da ação humana, mesmo quando seu objeto de estudo seja apenas um ponto específico de uma ação mais ampla (SCHWANDT, 2006). Assim, não é possível considerar que o pesquisador seja completamente neutro em seu campo de pesquisa. Portanto, compreende-se que os resultados advêm da interação entre pesquisador e pesquisados, e de suas escolhas no decorrer da pesquisa (escolhas de paradigma, de teoria norteadora, etc.).

Com isso, noto que, apesar de esforço para distanciar meus valores durante a condução da pesquisa, considero que não é possível a total supressão de aspectos pessoais neste processo. Portanto, julgo importante apresentar minhas preocupações de conduzir esta pesquisa de forma reflexiva. A reflexividade, considerada de alta importância por Altheide e Johnson (1994) e Cohen, Manion e Morrison (2000), aponta 
que o pesquisador deve reconhecer seu próprio eu dentro da pesquisa e que se mostrem cientes das maneiras como suas subjetividades podem influenciar o estudo. Por isso, é necessário que o pesquisador tenha a capacidade de também analisar suas ações, para compreender como elas fazem parte da pesquisa.

Em relação à metodologia, essa pesquisa qualitativa segue procedimentos de cunho etnográfico. A etnografia propõe o estudo de uma cultura ou, nesse caso, de um grupo escolar no qual o pesquisador se incorpora na comunidade a ser observada a fim de relatar suas observações das ações desse grupo ${ }^{3}$. Nesse sentido, a etnografia se coaduna com o interpretativismo, pois também pressupõe que o investigador descreva as ações observadas de maneira relativamente objetiva, de acordo com as intenções e significados dos atores (VIDICH; LYMAN, 2006).

Apesar da busca pela objetividade no relato dos resultados, recorro neste momento à instância epistemológica já discutida sobre a dificuldade da manutenção da neutralidade por parte do pesquisador. Vidich e Lyman (2006, p. 51) retratam os pesquisadores etnógrafos como

\footnotetext{
observadores do mundo, eles também participam deste; suas observações, portanto, são feitas dentro de um esquema mediado, ou seja, um esquema de símbolos e significados culturais oferecidos a eles por aspectos de suas histórias de vida que eles trazem para o ambiente observacional.
}

Outras características desta pesquisa que justificam essa classificação metodológica é que se realiza em ambiente natural, com objetivo de descrever e analisar conhecimentos e ações de determinado grupo de pessoas. Sendo importante também constatar que, para esta pesquisa, a situação observada é única, o que leva a cuidados em relação a generalizações, e que o processo e seu entendimento são partes centrais do estudo. (COHEN; MANION; MORRISON, 2000).

Para conseguir compreender as ações dos participantes e significados das mesmas para eles, recorro à observação participante, uma vez que ela permite que o pesquisador possa coletar dados por meio de ações verbais ou não verbais, conseguindo discernir comportamentos importantes para o caso estudados daqueles que não o são e contribui para o ambiente da pesquisa seja mais natural, sem interferências exteriores

\footnotetext{
${ }^{3}$ Apesar do grupo pesquisado neste estudo ter sido criado, considerei-o como pesquisa etnográfica pelo fato de eu estar integrada ao grupo, percebendo e descrevendo suas ações.
} 
que uma pesquisa normalmente impõe, visto que a interação com os participantes ocorreu em um período relativamente longo (COHEN; MANION; MORRISON, 2000).

\section{CONTEXTO DE COLETA E PARTICIPANTES}

O contexto no qual a coleta de dados aconteceu foi um curso de leitura em língua inglesa, com atividades elaboradas a partir dos pressupostos da teoria do contexto da situação (HALLIDAY, 1989), oferecido para alunos de ensino Médio (1º a $3^{\circ}$ ano) de uma escola pública de Londrina - PR. O curso foi realizado no contraturno, para que não influenciasse no andamento das aulas regulares da escola e para que os alunos que participaram do curso o fizessem com liberdade de escolha e vontade de fazerem parte do projeto. O tempo de duração do curso foi de 30 horas e foi ministrado pela professora-pesquisadora.

A escola onde o curso foi ofertado se localiza na região central do município de Londrina-PR, é de tamanho médio, possui 12 salas de aula, secretaria grande, salas para a coordenação e para a direção da escola, sala de professores, biblioteca, sala de informática, anfiteatro e duas quadras de esportes, sendo uma coberta, para as aulas de educação física. Durante o tempo que frequentei a escola, pude perceber o trabalho constante da coordenação e da direção com os alunos e no atendimento aos pais. Também conheci outros projetos que aconteciam naquele momento, como um projeto de confecção de um jornal pelos alunos e uma oficina de redação. Os alunos participantes das aulas deste projeto tinham entre 15 e 17 anos, de classe média, cursavam as séries do ensino Médio tanto do período matutino quanto vespertino.

A coleta de dados ocorreu de modo longitudinal, por meio das atividades aplicadas durante o curso. Para este trabalho, devido ao espaço reduzido, selecionei a participação dos alunos em uma das atividades de leitura realizadas durante as aulas. Com isso, o objetivo da análise desses dados é compreender processos de leitura dos alunos durante essa atividade, com foco em desenvolvimento (ou não) de leitura em nível crítico.

A atividade escolhida refere-se a um vídeo disponível na internet, no qual o ator Sean Connery faz um discurso em homenagem ao ator e cineasta Al Pacino, 
durante a entrega de um prêmio a este ${ }^{4}$ (apêndice). A escolha dessa atividade para ser analisada neste trabalho se deve ao fato de que ela não foi a primeira atividade de leitura a ser realizada no curso, assim, considero que os alunos estavam adaptados com o formato das questões ${ }^{5}$. Dentro dessa atividade utilizada como fonte de dados para este trabalho, optei por analisar algumas questões. Selecionei cinco questões de um total de nove da atividade mencionada. Essa seleção foi feita porque avaliei essas questões como proponentes de possível leitura em nível crítico ${ }^{6}$. Em relação aos alunos, apresento as respostas de oito participantes, os quais cursaram as aulas até o fim.

Para a análise das respostas dos alunos, avaliei-as como críticas ou não críticas. $\mathrm{Na}$ categoria de resposta não crítica, englobei respostas que não apresentaram questionamento sobre o texto/discurso. Nesse caso, o esperado desse tipo textual é que a pessoa que profere o discurso faça homenagem ao colega e, portanto, apresente ponderações que caracterizem ou se refiram ao homenageado de forma positiva. A aceitação, por parte do leitor, dessa maneira esperada de construção textual, sem problematização sobre o discurso em si, produz uma leitura em nível não crítica. Por outro lado, na categoria de resposta crítica, selecionei as respostas que apresentavam algum nível de questionamento sobre essa estrutura esperada do texto/discurso, não simplesmente o aceitando como uma homenagem, caracterizado positivamente, mas que, de alguma forma, demonstravam a interpretação do leitor em relação ao discurso em si, observando sua ironia.

\section{ANÁLISE DOS DADOS}

O total de respostas analisadas neste trabalho foi de 40 questões ( 5 questões de 8 respondentes). Em um resultado geral, esta análise totalizou 16 respostas não críticas, 16 respostas críticas, das quais 5 não apresentaram justificativa, 2 respostas foram consideradas parcialmente críticas e parcialmente não críticas e 6 não respostas. Neste trabalho, selecionei alguns exemplos para ilustrar cada categoria citada, comentando sobre sua classificação.

\footnotetext{
${ }^{4} \mathrm{O}$ vídeo está disponível no endereço eletrônico <http://www.youtube.com/watch?v=435ReudDn7I>.

5 A estrutura das questões causou estranhamento por parte dos alunos em primeiro momento, devido ao fato de não haver apenas uma resposta possivelmente correta e de que os alunos podiam assinalar quantas alternativas julgassem necessário.

${ }^{6}$ Avaliei as outras questões, que não são analisadas neste estudo, como proponentes de leitura em nível de interpretação.
} 
Como já apontado anteriormente, considerei como respostas não críticas as respostas que apresentam a aceitação do discurso, sem questionamento em relação às intenções do autor da fala. Apresento duas respostas como exemplos dessa categoria e assinalo as respostas dadas pelos alunos em itálico.

When Sean Connery says: "the AFI is different: we're all here to say "You're a winner, we appreciate you, and for all your marvelous performances that you've given over the years, we thank you. Congratulations", he:

a) reproduces social conventions

b) expresses his personal appreciation

c) he follows institutional rules

d) he subverts institutional rules

Escolhi a B porque Sean nessa parte fala bem de Al Pacino.

Sean Connery's speech is to Al Pacino:

\author{
a) an insult \\ b) a tribute \\ c) an attack \\ d) a praising \\ Sean Connery ['s] speech [is] a tribute cause [it] is [his] role in the honor.
}

Nessas respostas, compreendo que a interpretação dos alunos se relaciona com a aceitação do senso comum: o papel do ator Sean Connery é fazer um discurso de homenagem a Al Pacino e é essa a interpretação mais adequada. Não há questionamento sobre a fala do ator, se sua real intenção é homenagear Al Pacino ou se há outra intenção subentendida no discurso.

Julguei como respostas críticas aquelas que apresentam alguma forma de questionamento e não aceitação da interpretação mais condizente com o senso comum. Seguem dois exemplos:

In your opinion, how does Al Pacino feel while Sean Connery speaks?
a) happy
f) dissatisfied
b) sad
g) tense
c) comfortable
h) relaxed
d) uncomfortable
i) embarrassed
e) satisfied
j) disappointed

Why does he feel like that?

Ele se sente assim ao perceber que o Sean usou o sarcasmo para falar do Al Pacino. Eu descobri pelas caras e bocas do Al Pacino.

When Sean Connery says: "the AFI is different: we'll all here to say "You're a winner, we appreciate you, and for all your marvelous performances that you've given over the years, we thank you. Congratulations", he:

a) reproduces social conventions

b) expresses his personal appreciation

c) he follows institutional rules

d) he subverts institutional rules

Ele segue regras. A parte "we..." (nós), ou seja, a AFI, não o Sean, ou seja, ele foi um porta voz. 
Nessas respostas, observo questionamento das intenções de Sean Connery ao realizar sua fala, no sentido de subverter o senso comum de aceitar esse discurso como homenagem e perceber um possível ataque velado e ou ironia nas palavras do ator escocês. Acentuo a interpretação da imagem do ator Al Pacino na primeira questão, possibilitando ao aluno perceber o incômodo do ator americano durante a fala, e a análise do pronome nós (we), na segunda questão, levando o aluno a perceber a possibilidade de Sean não estar relatando sua opinião pessoal, mas sim falando em nome de um grupo de pessoas.

As duas respostas que avaliei como parcialmente críticas e parcialmente não críticas são as seguintes:

The tone of the speech is:
a) sincere
d) arrogant
b) ironic
e) humble
c) pedantic
c) pedantic
To Sean, [it] was a true speech, but to Al, seemed to be ironic.

In your opinion, how does Al Pacino feel while Sean Connery speaks?
a) happy
f) dissatisfied
b) sad
g) tense
c) comfortable
h) relaxed
d) uncomfortable
i) embarrassed
e) satisfied
j) disappointed

Why does he feel like that?

He felt happy cause he was laughing but tense cause Sean Connery was being pedantic.

Considerei a primeira resposta parcialmente crítica pelo fato do aluno perceber o incômodo de Al Pacino e possível ironia no discurso de Sean Connery. Apesar disso, o aluno não expressa questionamento e ou problematiza as intenções do autor do discurso, aceitando-o como sincero e verdadeiro. Na segunda questão, a parte da resposta que analisei como crítica foi a percepção, por meio das imagens, da tensão na expressão do cineasta americano por causa do discurso, mas avaliei a atribuição do sentimento de felicidade ao riso de $\mathrm{Al}$ Pacino como não crítica, pois o aluno não problematiza que a risada de do homenageado pode ser fingimento ou atribuída a um sentimento de nervosismo, ao invés de felicidade, como o senso comum pressuporia.

Por fim, apresento exemplos de não respostas e, na sequência, comento os motivos que considerei para essa classificação. 
When Sean Connery says: "the AFI is different: we're all here to say "You're a winner, we appreciate you, and for all your marvelous performances that you've given over the years, we thank you. Congratulations", he:

a) reproduces social conventions

b) expresses his personal appreciation

c) he follows institutional rules

d) he subverts institutional rules

Because he say[s] bad coisas sobre Al Pacino e só sobre ele (Sean).
a) reproduces social conventions
b) expresses his personal appreciation
c) he follows institutional rules
d) he subverts institutional rules
Because he [says] what he thinks of Al Pacino.

Apesar de a primeira resposta poder ser qualificada como crítica, considerei-a como não resposta por não estar relacionada com a pergunta. Nesse caso, o aluno comentou sobre o discurso/texto como um todo, e não sobre o trecho ao qual a pergunta se referia. Já a segunda resposta, avaliei-a como não resposta porque a justificativa dada pelo aluno parece não estar relacionada com a opção assinalada por ele.

\section{DISCUSSÃO DOS RESULTADOS}

A partir da análise das respostas dos alunos às questões de uma atividade de leitura conduzida em curso de leitura em língua inglesa, considero que, mesmo totalizando metade de respostas críticas e metade de respostas não críticas, há resultados que podem ser considerados positivos, visto que os alunos que demonstraram práticas de leitura em nível crítico são exemplos da possibilidade de trabalho com leitura crítica nas aulas de língua inglesa e de seus bons resultados. Além disso, as respostas que avaliei como não críticas também apresentam bom nível de reflexão e interpretação dos alunos, mesmo eles não alcançando nível crítico de leitura.

Considerando que, devido à falta de familiaridade que percebi por parte dos alunos com atividades de leitura em língua inglesa como as desenvolvidas durante o curso, as respostas avaliadas como críticas são bons indícios da capacidade de desenvolvimento de consciência crítica com os alunos em aulas de língua estrangeira, a partir de trabalho pautado em leitura crítica. 


\section{CONSIDERAÇÕES FINAIS}

Neste estudo, analisei respostas de alunos do ensino Médio em atividade de leitura realizada durante curso de leitura em língua inglesa conduzido por mim em escola pública de Londrina - PR. A atividade de leitura foi produzida a partir dos pressupostos da teoria do contexto da situação (HALLIDAY, 1989), como meio de proporcionar leitura crítica aos alunos. Nesta análise, meu objetivo foi descrever as práticas de leitura desses alunos nessa atividade, com prioridade em notar respostas críticas produzidas por eles.

Em primeiro lugar, considero de grande valia formas de trabalho docente que fujam do recorrente ensino de vocabulário e regras gramaticais observado nas escolas. Mais que isso, propiciar trabalho pedagógico que ajude a construir nos alunos uma visão mais crítica da sociedade e das informações que recebem todos os dias é caminhar ao encontro com objetivos educacionais e sociais de formação cidadã apontados pelas OCEM (BRASIL, 2006). Assim, avalio que leitura crítica como embasamento de aulas de leitura em língua estrangeira apresentou bons resultados em minha experiência e tem grande valor. Contudo, é sempre importante considerar que meu objetivo não é defender tal teoria como melhor forma de ensino de língua inglesa, uma vez que há inúmeras metodologias de ensino que contribuem para o trabalho docente e o professor efetua suas escolhas de acordo com seus objetivos em sala de aula.

Assim, finalizo observando que minha experiência de leitura crítica em sala de aula de língua inglesa contribuiu para meu entendimento a respeito da prática docente embasada nessa teoria e que os resultados deste trabalho demonstram boas perspectivas para trabalho pedagógico que vise a construir consciência crítica com os alunos e contribuir para sua formação cidadã.

\section{REFERÊNCIAS}

ALTHEIDE, D.; JOHNSON, J. Criteria for assessing interpretive validity in qualitative research. In: DENZIN, N.; LINCOLN, Y. (Eds.). Handbook of qualitative research. London: Sage Publications, 1994. p. 485 - 499.

BRASIL. Ministério da Educação. Secretaria de Educação Básica. Conhecimentos de Línguas Estrangeiras. In: BRASIL. Ministério da Educação. Secretaria de Educação 
Básica. Orientações Curriculares para o Ensino Médio (OCEM): Linguagens, Códigos e suas tecnologias. Brasília, 2006, v. 1.

COHEN, L.; MANION, L.; MORRISON, K. Research methods in education. London; New York: Routledge, 2000d.

DECHANT, E. The reading process. In: Understanding and teaching reading: an interactive model. New Jersey: Lawrence Erlbaum, 1991. p. 1 - 37.

FAIRCLOUGH, N. Language and power. London e New York: Longman, 1989.

Global capitalism and critical awareness of language. 1999. Disponível em: <http://eprints.lancs.ac.uk/8546/1/la0080071.pdf>. Acesso em 20 de maio de 2012.

FIGUEIREDO, D. C. Critical discourse analysis: towards a new perspective of EFL reading. Ilha do Desterro, n. 38, p. 139 - 154. jan./jun. 2000.

GUBA E. The alternative paradigm dialog. In: GUBA, E. (Ed.). The paradigm dialog. Newbury Park, CA: Sage Publications, 1990.

HALliDAY, M. A. K. Part A. In: HALliDAY, M. A. K.; HASAN, R. Language, context and text: aspects of language in a social-semiotic perspective. Oxford: Orford university Press, 1989. p. $3-49$.

HARRISON, C. What does research tell us about the reading process and the early stages of reading development? In:

London: Sage Publications, 2004. p. $26-49$.

Understanding reading development.

SCHWANDT, T. A. Três posturas epistemológicas para investigação qualitativa: interpretativismo, hermenêutica e construcionismo social. In: DENZIN, N. K.; LINCOLN, Y. S. (Eds.). O planejamento da pesquisa qualitativa: teorias e abordagens. Porto Alegre: Artmed, 2006. p. 193-217.

TAGLIEBER, L. K. Critical reading and critical thinking: the state of art. Ilha do Desterro, Florianópolis, n. 38, p. 15-37. jan./jun. 2000.

TOMITCH, L. M. B. Designing reading tasks to foster critical thinking. Ilha do Desterro, Florianópolis, n. 38, p. 83 - 90. jan./jul. 2000.

VIDICH, A.; LYMAN, S. M. Métodos qualitativos: sua história na sociologia e na antropologia. In: DENZIN, N. K.; LINCOLN, Y. S. (Eds.). O planejamento da pesquisa qualitativa: teorias e abordagens. Porto Alegre: Artmed, 2006. p. 49-90.

WALLACE, C. Critical literacy awareness in the EFL classroom. In: FAIRCLOUGH, N. (Ed.). Critical Language Awareness. London: Longman, 1992. p. 59 - 92. 


\section{APÊNDICE - ATIVIDADE DE LEITURA}

Introdução: As atividades propostas a seguir foram elaboradas a partir de um vídeo no qual o ator Sean Connery faz um discurso homenageando Al Pacino, na ocasião do recebimento do seu prêmio da AFI (American Film Institute). O objetivo das atividades é conduzir os alunos a analisarem o texto / discurso criticamente, observando intenções implícitas que podem ser desvendadas por meio da linguagem e dos recursos visuais.

\section{Atividade: Vídeo de Sean Connery homenageando Al Pacino em prêmio da AFI.}

\section{Script do vídeo:}

Announcer: Ladies and gentlemen, the 2006 recipient of the AFI achievement award: Sean Connery.

Sean Connery: I know what some of you are thinking: "What the hell is he doing back here?" But...my dear Al, we've known each other for 30 years and never worked together, which was my loss. However, to share the AFI Award makes for another marvelous bond. I sat in that chair last year, and I know how it feels. There's nowhere to hide. You'll remember many good times and some dodgy times. It's a humbling experience, uh?, for you look at those people and might wonder if you belong. Well, Al, you belong. My advice for you is simple: "Enjoy" - for in this business that likes to put us against each other as winners and losers - well, the AFI is different: we're all here to say "You're a winner, we appreciate you, and for all your marvelous performances that you've given over the years, we thank you. Congratulations".

Source: http://www.youtube.com/watch?v=435ReudDn7I

1. Watch the video and complete the speech:

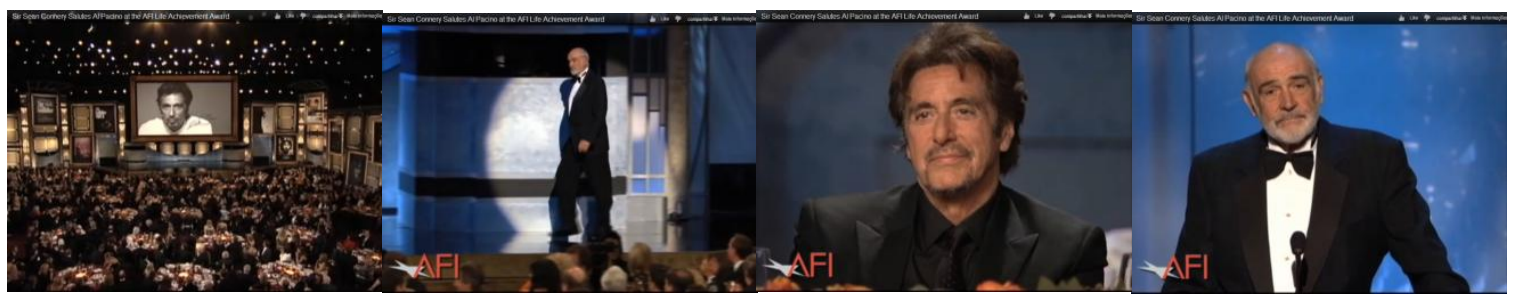

Announcer: Ladies and gentlemen, the 2006 recipient of the AFI achievement award: Sean Connery.

Sean Connery: I know what some of you are thinking: "What the hell is he doing back here?" But...my dear Al, we've known each other for years and worked which was my bond. I sat in that chair last year,

AFI Award makes for another . You'll remember many and I know how it feels. There's nowhere to times. It's a humbling experience, uh?, for times and some Well, Al, you you look at those people and might wonder if you My advice for you is simple: " $"$ - for in this business 
that likes to put us each other as winners and - well, the AFI is different: we're all here to say "You're a we appreciate you, and for all your marvelous performances that you've given over the years, we thank you. Congratulations".

2. Tick ( V ) the points in the transcription when applause occurs.

3. Who is more mentioned in the speech, Al Pacino or Sean Connery?

4. Highlight the positive parts about Al Pacino in the speech.

5. How can you define the situation presented in the video? Mark (T) true or (F) false and, for the false ones, explain why you considered them false.

a) ( ) it's a public event in which a person is being honored.

b) ( ) the level of formality of the occasion is low and it's not necessary to care a lot about the language.

c) ( ) it's a local event and few people will be able to access it.

d) ( ) it belongs to the work sphere because the participants work in the same area.

6. What is the relationship between the participants of the video?

a) They are friends.

b) They are work colleagues.

c) They are acquaintances.

d) any other kind of relationship. What?

7. In the situation, is anybody in a higher position than the other? Why?

8. In your opinion, how does Al Pacino feel while Sean Connery speaks?
a) happy
f) dissatisfied
b) sad
g) tense
c) comfortable
h) relaxed
d) uncomfortable
i) embarrassed
e) satisfied
j) disappointed

Why does he feel like that?

Comments:

9. When Sean Connery says "You'll remember many good times and some dodgy times. It's a humbling experience, uh?", what is his intention?

a) to anticipate that A1 Pacino's situation is comfortable

b) to anticipate that Al Pacino's situation is uncomfortable

c) to insinuate that $\mathrm{Al}$ Pacino will have to rethink what he did in the past

Comments: 
10. Sean Connery's speech is to Al Pacino:

( ) an insult

( ) an attack

( ) a tribute

( ) a praising

Comments:

11. The tone of the speech is:
( ) sincere
( ) ironic
( ) arrogant
) pedantic
( ) humble

Comments:

12. When Sean Connery says: "the AFI is different: we'll all here to say "You're a winner, we appreciate you, and for all your marvelous performances that you've given over the years, we thank you. Congratulations", he:
( ) reproduces social conventions
( ) expresses his personal appreciation
( ) he follows institutional rules
( ) he subverts institutional rules

Comments: 